\title{
Introduction to the special issue
}

\author{
Jamie Davies $\cdot$ Michael Grinfeld $\cdot$ Steven D. Webb
}

Published online: 27 August 2010

(C) Springer-Verlag 2010

This special issue of Theory of Biosciences is the response to call for papers we circulated during and following the International Centre for Mathematical Sciences (ICMS) workshop on Emerging Modelling Methodologies in Medicine and Biology, held in Edinburgh July 20th-July 24th, 2009.

The aim of the meeting was to bring together modellers (mathematicians, physicists, computer-scientists, or engineers) attracted by the challenges and opportunities provided by twenty-first century life sciences, biologists with a synoptic view of their disciplines looking for over-arching explanatory schemes, and philosophers of science interested in the nature of biological systems, explanation, and understanding in biology.

We thought that organising such a meeting was timely and important, because from the point of view of biology, we live in exciting times. New techniques for the investigation of life at the subcellular level appear all the time, and new data on the organisation and function of macromolecules are continuously collected. In the resulting increasingly and recursively involved picture, we need to uncover principles that govern this complexity. In many areas of science, notably in physics, mathematics has been successfully used to deal with complexity. The overarching

\footnotetext{
J. Davies

Centre for Integrative Physiology,

University of Edinburgh, Edinburgh, Scotland

e-mail: jamie.davies@ed.ac.uk

M. Grinfeld · S. D. Webb $(\bowtie)$

Department of Mathematics and Statistics,

University of Strathclyde, Glasgow, Scotland

e-mail: steven.webb@strath.ac.uk

M. Grinfeld

e-mail: m.grinfeld@strath.ac.uk
}

aim of the meeting was to discuss foundational methodologies of the "mathematisation of biology" (Israel 1996). There is limited methodological work on modelling in biology; see for example (Fox-Keller 2000; Lander 2004), or the cautionary work in Buiatti (1998) and Rosen (1985).

In biology, unlike physics, there are no discernible natural laws to anchor modeling to. In addition, biological systems are distinguished by being the product of evolutionary forces, and they persist in time while recycling and renewing their components. In biology it is very rare to have a system of a few interacting parts on the same scale: biological systems are complex, multi-scale systems by definition. Hence modelling a biological system is a highly nontrivial task. At the same time, mathematical formalism seems to be the only approach for dealing with the complexity of biological systems.

There is a real need to confront this complexity, since in therapy, in principled drug design, or in ecosystem management it is often necessary to predict what the system as a whole would do in particular circumstances. The piecemeal methodology of experimental biology has no tools of its own to address this need.

Complex systems, that is systems comprised of many tightly coupled heterogeneous (by nature and scale) components, can be approached in a number of ways. The reductionist way of an engineer is to decompose the system into simpler subsystems, analyse the workings of each subsystem separately, understand the coupling of the subsystems, and reconstruct in this way the function of the entire "mechanism." This approach does not work in biology as it is not clear how, even in principle, to define a (reasonably closed) subsystem. So far, reductionist approaches in biology always leave open an explanatory gap between what the system does and what can with confidence be said about its parts. 
From the above remarks, the usefulness of a multidisciplinary approach to modelling in the life sciences should be clear. In the event, we had a very enthusiastic response from the modelling community and a strong showing from biologists in areas where some form of holistic approach is seen as necessary (ageing, cancer, developmental biology, structure of the nervous system). Unfortunately, for administrative reasons, we were not able to recruit as many philosophers of science as we would have wanted, and furthermore, all accepted papers in this special issue come from the modelling community.

It seems to us that the set of fundamental questions we have identified (see http://www.icms.org.uk/workshops/ modellingmethodologies) is acutely relevant for developing successful applications of mathematical modelling in biology and medicine, and we hope that future meetings will realise our vision of a philosophically and biologically informed modelling activity.

This special issue thus covers a variety of modelling approaches, encompassing both traditional techniques and a range of emerging methodologies. Differential equations form the main theme but these are approached, derived, and indeed used from a number of different angles. Examples include their application to a problem of energy metabolism at the whole organismal scale, their rigorous derivation using process algebra techniques, their coupling to bioinformatic data to provide a multifactorial network approach to model protein-protein interactions and their applications to evolutionary theory at the molecular level. Additionally, alternatives to differential equations are considered, such as stochastic branching processes and stochastic molecular simulations; the fast growing area of systems biology mark-up languages is also discussed. A summary of the contents of this special issue is as follows:

Göbel and Langemann (2010) create a differential equation model to study the short- and long-term behaviour of the body in terms of its appetite regulation and energy use. A central and novel concept is the 'Selfish Brain' theory, which includes regulatory mechanisms of both the glucostatic and the lipostatic theory, and extends them by the administrative position of the brain in the regulatory hierarchy.

McCaig et al. (2010) introduce process algebra techniques to the field of epidemiology. This approach from computer science allows the system to be initially described in terms of stochastic processes at the level of an individual, and then be scaled up to explore the dynamics of an entire population. The process output is usually defined in terms of differential equations which can then be solved using traditional techniques. In this article, the authors focus on a rigorous derivation of a disease transmission model by considering individual-level stochastic epidemiological interactions, with the key ingredients being transmission of infection and acquired immunity. This article represents a thorough and neat introduction to, and an overview of the concepts behind process algebra and its utility in modelling biological systems.

MacMillan and McConnell (2010) develop branching process models to account for cell type heterogeneity in cerebral cortex development, highlights the advantages of this approach over averaged-level models. The analysis proceeds from considering the case of one proliferative progenitor cell type to the problem of four cell types, in which the roles of separate apical and basal progenitors are considered.

Ruebenacker and Blinov (2010) describe how large web-based data sets (referred to as the Systems Biology 'Cloud') can be processed efficiently into annotated computational models. The key elements of this conversion are taken to be: (1) the representation of processes (e.g., sets of biological reactions) and substances (e.g., sets of biological specimens) and (2) the grouping of entities into 'views' (e.g., a bipartite graph representing the interactions concerned). Different aspects of these key elements as well as manipulation tools are discussed, and the advantages in comparison to other existing mark-up languages of a specific Java application (SyBil) are highlighted.

Nikolov et al. (2010) present and discuss a differential equation approach to link miRNA translational control with cell signaling networks. Data on protein-protein interactions and miRNA regulation obtained from bioinformatics databases are integrated with the multifactorial network modeling approach and an exciting application to colorectal cancer, in terms of the progression of several tumour subtypes, is discussed and analyzed.

Finally, the article by Schuster (2010) presents a summary of the status of mathematical modelling of evolution at the molecular level. He begins with classical models of selection resulting from competition under logistic growth. The article then goes on to discuss how combining this theory with Mendelian inheritance leads to population genetics; molecular evolution is introduced and an approach to model it as a process of replication and mutation is presented. Error thresholds, fitness landscapes, and genotype-to-phenotype maps are discussed. Finally, the existence of neutral sets of genotypes and the necessity for stochastic molecular simulation approaches are considered.

Acknowledgments We are grateful to ICMS (Edinburgh), LMS, BTG (University of Strathclyde), and CSBE (University of Edinburgh) for their funding of the workshop, to the ICMS staff for the expert handling of all the aspects of the workshop administration, to Professor J. Jost for allowing us to put together papers stemming from the workshop as a special issue of Theory in Biosciences and for carefully reading the submissions, and to each other for good-natured collaboration. 


\section{References}

Buiatti M (1998) Mathematical modelling in biology: a critical assessment. Il Nuovo Cimento 20:79-89

Fox-Keller E. (2000) Models of and models for: theory and practice in contemporary biology. Phil. Sci. 67:S72-S86

Göbel B, Langemann D (2010) Systemic investigation of a braincentered model of the human energy metabolism. Theory Biosci. doi:10.1007/s12064-010-0105-9

Israel G (1996) Mathématisation du réel. Seuil, Paris

Lander AD (2004) A calculus of purpose. PLoS Biol 2:0712-0714

MacMillan HR, McConnell MJ (2010) Seeing beyond the average cell: branching process models of cell proliferation, differentiation, and death during mouse brain development. Theory Biosci. doi:10.1007/s12064-010-0107-7
McCaig C, Begon M, Norman R, Shankland C (2010) A rigorous approach to investigating common assumptions about disease transmission. Theory Biosci. doi:10.1007/s12064-010-0106-8

Nikolov S, Vera J, Schmitz U, Wolkenhauer (2010) A model-based strategy to investigate the role of microRNA regulation in cancer signalling networks. Theory Biosci. doi:10.1007/s12064-0100109-5

Rosen R (1985) Organisms as causal systems that are not mechanisms: an essay on the nature of complexity. In: Rosen R (eds) Theoretical biology and complexity. Academic Press, New York Ruebenacker O, Blinov M (2010) Using views of Systems Biology Cloud: application for model building. Theory Biosci. doi: 10.1007/s12064-010-0108-6

Schuster P (2010) Mathematical modeling of evolution solved and open problems. Theory Biosci. doi:10.1007/s12064-010-0110-z 